\title{
Modulations of emotional attention and spatial attention on human visual cortical activities
}

This article was published in the following Dove Press journal:

Psychology Research and Behavior Management

Taiyong $\mathrm{Bi}^{1, *}$
Yangyang $\mathrm{Du}^{2, *}$
Xiaogang Wang
Na Sang'
Fan Zhang
Hui Kou'
Qian Zhu'
Jiang Qiu ${ }^{2}$
'School of Management, Zunyi Medical
University, Zunyi, People's Republic of
China; ${ }^{2}$ Key Laboratory of Cognition and
Personality (SWU), Ministry of Education,
Chongqing, People's Republic of China
*These authors contributed equally to
this work

*These authors contributed equally to
this work
Correspondence: Taiyong $\mathrm{Bi}$

School of Management, Zunyi Medical

University, No. 6 Xuefu West Road,

Xinpu, Zunyi, Guizhou 563000, People's

Republic of China

Tel +86I 8696850920

Email bitaiyong@126.com

Jiang Qiu

Faculty of Psychology, Southwest

University, No. 2 Tiansheng Road, Beibei,

Chongqing 400715, People's Republic of

China

Tel +86I 3883840095

Email qiuj318@swu.edu.cn
Background: Spatial attention could enhance the neural activities of attended locations in human visual cortex. As a salient stimulus, emotional image could enhance the responses in amygdala and visual areas. However, few studies examined the interaction of the attentional effects induced by emotional stimuli and spatial cues on the neural responses in visual areas. Methods: In the present study, we used functional magnetic resonance imaging (fMRI) to examine the combined and separated effects of emotional image and spatial cue on the activities in human visual areas. A revised Posner cueing paradigm was utilized. Each participant viewed a fearful image and a peaceful image simultaneously in left and right visual fields. A spatial cue of two dots was then presented in one of the image positions. In this manner, the attentional effects for emotional image and spatial cue could be isolated and combined.

Results: The results showed that spatial cue enhanced the responses in V4, intraparietal sulcus (IPS) and lateral occipital area (LO), while emotional image could enhance the responses in V3, V4 and LO. Importantly, no significant interactions were found in any of the visual areas.

Conclusion: Our results indicate that the two kinds of attentional modulation might not be affected by each other. These findings shed light on the neural mechanism of the emotional attention.

Keywords: emotional attention, spatial attention, visual cortex, fMRI

\section{Introduction}

Spatial selective attention means that the perception of a stimulus at the cued location can be improved significantly, as indicated by higher accurate rates and shorter reaction times. ${ }^{1,2}$ The cueing paradigm is usually termed Posner cueing paradigm. Using such a paradigm, neurophysiological studies show that neural responses are not only found to be increased when attention was paid to the cued location, ${ }^{3-5}$ but also on salient stimuli. ${ }^{6-9}$

Previous studies using Posner cuing paradigms found that the neural correlates of spatial selective attention consist of a dorsal frontoparietal attentional network (DAN) and a ventral attentional network (VAN), ${ }^{10,11}$ and both of them need the involvement of visual cortical activity. ${ }^{12}$ Frontoparietal cortex is shown to be a core structure in cognitive functions such as attentional control and working memory. ${ }^{13,14}$ Attentional modulation from the frontoparietal areas to visual cortex is essential for the selective attention while selecting visual locations. ${ }^{12,15,16}$ In addition to top-down modulation, salient stimulus could also boost the activities in the visual cortex through an automatic bottom-up process. ${ }^{17-21}$ 
One kind of salient stimuli that can lead to automatic, involuntary attentional bias are images with emotional content, such as fearful faces. ${ }^{22}$ Such an attentional bias was termed as emotional attention. ${ }^{22}$ The core neural structure underlying emotion processing is amygdala. ${ }^{22-25}$ Amygdala not only monitored the emotional value, but also projected to prefrontal areas and striate and extra-striate visual cortex to regulate appropriate reactions. ${ }^{26}$ Neural projection from the amygdala to occipital cortex might be involved in improving visual perception of emotionally salient stimuli. ${ }^{18}$ In addition, studies also found enhanced functional connectivity between amygdala and inferior temporal cortex while processing emotional stimuli. ${ }^{27,28}$

Although spatial attention and emotional attention both could enhance the visual processing and neural activity corresponding to the cued location, they are two kinds of attention with different sources and neural pathways. It is still elusive that whether the two kinds of attentional modulation interact with each other. Behavioral studies utilizing Posner cueing paradigm found that the performance in cued spatial locations could be further improved when the cued stimuli was emotion-laden stimuli. ${ }^{20}$ Consistently, using functional magnetic resonance imaging (fMRI) methods, researchers have found an additive modulation of spatial attention and emotional attention in amygdala and fusiform face area. ${ }^{29,30}$ However, these results did not indicate the interaction effect between the two kinds of attention. Eventrelated potentials (ERPs) studies found that early ERPs component are sensitive to emotional content, followed by a spatial attention sensitive component. ${ }^{19,31}$ These results demonstrated two independent modulations on different ERPs components, suggested no interaction between spatial and emotional modulations. However, when the gaze was adopted as the spatial cue, ERPs study showed an interaction effect of expression and gaze on N1 component. ${ }^{32}$ In summary, there is no conclusion on the interaction effect of spatial and emotional attention.

Previous studies revealed that spatial attention and emotional attention both influence the activities in human visual cortex. In the present study, we aim to investigate whether these two kinds of attentional modulation interact with each other. In order to address this problem, we utilized a modified Posner cueing paradigm with fMRI scanning. We separately manipulated the spatial attention and emotional attention. Functional MRI analysis may help us observe the isolated effects of emotional attention and spatial attention as well as a combined effect of these two kinds of attention in each visual area.

\section{Material and methods}

\section{Participants}

In total, 17 naïve healthy individuals ( 9 female, $M=20.0$ years old, $\mathrm{SD}=1.4$ ) participated in our experiment. Participants were from Southwest University, China. All participants were right-handed, with no history of neurological or psychiatric problems. Written informed consents were provided by all participants prior to the study, which was approved by the Institutional Human Participants Review Board, Imaging Center for Brain Research, Southwest University. This study was conducted in accordance with the Declaration of Helsinki. One subject was excluded due to excessive head motion in the scanner, resulting in 16 valid subjects in fMRI data analysis.

\section{Materials}

Original images consisted of 20 fearful and 20 peaceful pictures taken from the International Affective Picture System (IAPS). Fearful pictures include threatening contents such as bugs, snakes or skulls. Peaceful pictures include nonthreatening animals such as birds or butterflies. Subjects were asked to rate their fearful feelings of these selected IAPS pictures by a 5-point scale, from number 1 "not fearful at all" to 5 "very fearful". Based on the averaged rating scores, the 10 lowest scored peaceful pictures and the 10 highest scored fearful pictures were selected and presented pairwise during the experiment. The size of each image was $3.2^{\circ} \times 4.2^{\circ}$. The spatial cue was two tiny solid dots with diameters of $0.1^{\circ}$ and the distance between them was $0.5^{\circ}$, which were arranged horizontally or vertically. A cross always appeared on the center of the screen as a fixation. The distance from the center of the image or of the spatial cue to the fixation point was $6.3^{\circ}$.

\section{Experimental procedure}

As shown in Figure 1, subjects were asked to pay attention to the fixation center during the whole experiment. Each trial started with two simultaneously presented pictures which appeared for $50 \mathrm{~ms}$ in both sides of the visual field. One of the pictures was a fearful picture. The other was a peaceful one. Pictures were randomly selected from the fearful and peaceful pictures. After a $250 \mathrm{~ms}$ blank interval, a two-dot probe appeared for $100 \mathrm{~ms}$ in left or right visual fields. Subjects were asked to press keys to indicate the orientation of the probe (ie, ' 1 ' for horizontal orientation and ' 2 ' for vertical orientation) as accurately 


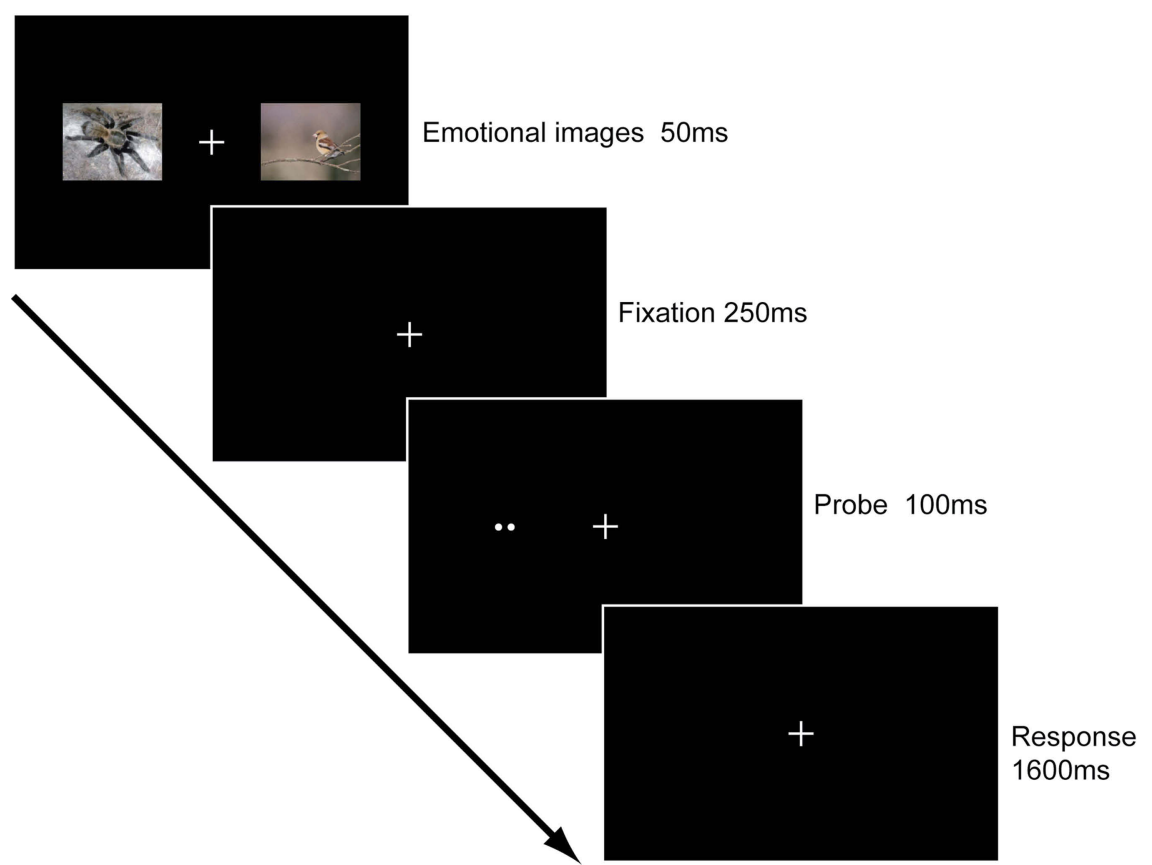

Figure I Schematic description of the stimulus pattern of one trial in the main experiment. Each trial began with two images in the left and right visual fields. One image was a threatening picture and the other was a neutral picture. After a blank interval, a spatial cue was presented in either the left or the right visual fields. Subjects were required to report the orientation of the spatial cue within I,600 ms.

and quickly as possible in a 1,600 ms blank interval. Therefore, each trial lasted for $2 \mathrm{~s}$. Intervals between two sequential trials were pseudorandomly selected from 2, 4 or $6 \mathrm{~s}$, resulting in an average inter-trial interval of $4 \mathrm{~s}$. There were 192 pairwise pictures in total, which was divided into 6 runs. Each run consisted of 32 trials, with a 12-s blank interval at the beginning and another one at the ending of the run. The positions of emotional pictures and probes were counterbalanced in each run, resulting in same trial numbers (48 trials) for following four conditions: left fearful picture \& left spatial cue, left fearful picture \& right spatial cue, right fearful picture \& left spatial cue and right fearful picture \& right spatial cue.

\section{Retinotopic imaging}

Retinotopic visual areas (V1, V2, V3 and V4) were defined by a standard phase-encoded method, ${ }^{3,34}$ in which subjects viewed rotating wedge and expanding ring stimuli that created traveling waves of neural activity in visual cortex. More detailed description of the procedure could be found in previous studies. ${ }^{35-37}$ A blockdesign scan was used to define the regions of interest (ROIs), including object-selective areas (LO) and responsive areas in V1, V2, V3, V4 and IPS. Subjects viewed images of fearful pictures, neutral objects and texture patterns (scrambled fear picture), which had the same size and same location as the stimuli used in the main experiment. Images appeared at a rate of $2 \mathrm{~Hz}$ in a block of $12 \mathrm{~s}$, interleaved with a blank block of $12 \mathrm{~s}$. Each image was presented for $300 \mathrm{~ms}$, followed by a $200 \mathrm{~ms}$ blank interval. Each block type was repeated 5 times in the scan, which lasted $360 \mathrm{~s}$ in total. As a result, each block contained 24 trials, and each condition contained a total of 120 trials in this experiment. Subjects performed a oneback task during scanning.

\section{MRI data acquisition}

In the scanner, the stimuli were back-projected via a video projector (refresh rate, $60 \mathrm{~Hz}$; spatial resolution, $1,024 \times 768$ ) onto a translucent screen placed inside the scanner bore. Subjects viewed the stimuli through a mirror mounted within head cap. The viewing distance was $83 \mathrm{~cm}$. Functional MRI data were collected using a 3T Siemens Trio scanner with a 12-channel phase-array coil. BOLD signals were measured with an echo planar imaging sequence (echo time, $30 \mathrm{~ms}$; repetition time, 2,000 ms; field of view, 196×196 $\mathrm{mm}^{2}$; matrix, 64×64; flip angle, 90; slice thickness, $3 \mathrm{~mm}$; gap, $0 \mathrm{~mm}$; number of slices, 33; slice orientation, axial). The bottom slice was positioned at the bottom of the temporal lobes. A high-resolution 3D structural dataset (3D magnetization-prepared rapid acquisition gradient echo; $1 \times 1 \times 1 \mathrm{~mm}^{3}$ resolution) was collected 
in the same session before the functional imaging started. Subjects underwent two sessions, one for the retinotopic mapping and the other for the main experiment.

\section{MRI data processing and analysis}

The anatomical volume of each subject in the retinotopic mapping session was transformed into the AC-PC (anterior commissure-posterior commissure) space and then was inflated using Brain Voyager QX (Brain Innovation). Functional volumes in all the sessions of each subject were preprocessed, including 3D motion correction, linear trend removal and high-pass $(0.015 \mathrm{~Hz})$ filtering. ${ }^{38}$ Head motion was constrained at no more than $3 \mathrm{~mm}$ in any scanning session. The images were aligned to the anatomical volume of obtained retinotopic MRI mapping and were also transformed into the AC-PC space. The first 6 seconds of BOLD signals was discarded in order to minimize transient magnetic saturation effects. The ROIs in V1, V2, V3, V4 and IPS were defined as areas that responded more intensely to the textured patterns (scrambled picture) than blank interval $\left(P<10^{-8}\right.$, uncorrected) and confined by the V1/V2/V3/V4/IPS boundaries defined by the retinotopic mapping scan. LO was defined as the area that responded more intensely to the intact images than the scrambled images in the occipital-temporal cortex. The averaged Talairach coordinates were provided in Table 1.

For each visual field (left or right), there were four conditions of stimulus combinations. For example, for the left visual field, in one quarter of trials, the fearful picture was first presented in this visual field and then the spatial cue was also presented here. Similarly, in another quarter of trials, the

Table I Averaged Talairach coordinates for each ROI in each hemisphere

\begin{tabular}{|l|l|l|l|l|}
\hline \multicolumn{2}{|l|}{ ROIs } & X & y & z \\
\hline Left hemisphere & VI & -11.7 & -89.6 & -5.45 \\
& V2 & -17.1 & -87.3 & -4.1 \\
& V3 & -21.8 & -83.1 & -3.2 \\
& V4 & -27.7 & -71.8 & -11.9 \\
& LO & -41.0 & -70.0 & -3.3 \\
Right hemisphere & IPS & -24.4 & -74.1 & 28.4 \\
& VI & 10.6 & -87.8 & -2.7 \\
& V2 & I5.6 & -83.9 & -3.3 \\
& V3 & 20.7 & -79.5 & -3.7 \\
& V4 & 26.3 & -70.8 & -9.8 \\
& LO & 40.9 & -66.5 & -7.2 \\
& IPS & 25.9 & -73.7 & 30.5 \\
\hline
\end{tabular}

Note: Data were averaged from all the subjects. fearful picture was presented in this visual field but the spatial cue was not presented here. Therefore, for each visual field, the presentation of stimuli could be one of following four situations: spatial cue is congruent to fearful picture (both are presented or not presented); spatial cue is incongruent to fearful picture (only spatial cue or fearful picture is presented). According to the retionotopic projection from visual field to visual cortex, the information of a visual field projects to the contralateral hemisphere. Therefore, for each visual area in each hemisphere, there were also four conditions of stimulus presentation: contralateral to fearful picture \& spatial cue, contralateral to fearful picture $\&$ no spatial cue, contralateral to neutral picture \& spatial cue and contralateral to neutral picture $\&$ no spatial cue. We marked these four conditions as E\&S (contralateral to emotional picture \& spatial cue), E\&NS (contralateral to emotional picture \& no spatial cue), NE\&S (contralateral to neutral picture \& spatial cue) and NE\&NS (contralateral to neutral picture \& no spatial cue). The setting of the conditions is demonstrated in Figure S1A.

The event-related BOLD signals were calculated through custom MATLAB (www.mathworks.com) code separately for each subject, as a method used by Kourtzi and Kanwhisher. ${ }^{39}$ BOLD signal intensity was first extracted from predefined ROIs in left and right hemispheres of all the subjects by averaging the data from all voxels within the ROIs. Then event-related averaging was applied for each condition and each hemisphere of the ROIs. Next, we averaged the signals of the same condition in both hemispheres. Therefore, we finally obtained the signals for each condition and each ROI of V1/V2/V3/V4/IPS/LO. The BOLD signal change was the normalized BOLD signal relative to the mean intensity of a run in each ROI. For each condition, the activation was defined as the response of the third TR (4-6 seconds) of each trial after stimulus onset, which covered the peak of the hemodynamic response function (HRF) in human visual areas. The procedure of data analysis is demonstrated in Figure S1B.

\section{Experimental design and statistical analysis}

In each ROI, there were four conditions: $E \& S, E \& N S$, NE\&S and NE\&NS. The experimental design was a 2 (emotional attention: E/NE) $\times 2$ (spatial attention: S/NS) within subject design. SPSS 16.0 software was used for data analysis. Repeated measures analysis of variance (ANOVA) was performed for each ROI. Paired sample $t$ tests with Bonferroni adjustment were performed when 
planned comparisons were needed. The effect size (ES) was provided with each statistical test. The ES was the partial eta squared $\left(\eta^{2}\right)$ for the interaction effect and the main effect in ANOVA. In $t$-test, the ES was the standardized mean difference (Cohen's d).

\section{Results}

Subjects showed good performance in detecting the orientation of the spatial cue, at a level of nearly or more than $90 \%$ accuracy for each subject (range: $89.5-99.8 \%$; M $(\mathrm{SD})=96.1 \%(2.4 \%))$, indicating that they were concentrated on the current task. Then we extracted the BOLD signal change of each condition (E\&S, E\&NS, NE\&S and $\mathrm{NE} \& N S)$ in each ROI. A $2(\mathrm{E} / \mathrm{NE}) \times 2(\mathrm{~S} / \mathrm{NS})$ repeated measures ANOVA was used for analysis.

As shown in Table 2 and Figure 2, we did not find significant interaction effect between emotional and spatial attentional modulations in any of the defined ROIs. However, we found that the main effect of emotional cue was significant in V3, V4 and LO (all $P_{S}<0.002$ ), and that the main effect of the spatial cue was significant in V4, IPS and LO (all $P s<0.001$ ). Interestingly, in V4 and LO, we found significant main effects for both emotional and spatial cues, indicating that responses in these areas were both modulated by emotional attention and spatial attention. Yet no significant interaction effects were found in these areas, indicating that the modulations of the two kinds of attention weakly interacted with each other.

To further reveal the attentional modulations in each ROI, we compared the activations in each two conditions with paired sample $t$-test. First, V3 showed a significant main effect of emotional attention. However, we did not find any significant difference between any two conditions (all $P s>0.05$, Bonferroni corrected). Second, IPS showed a significant main effect of spatial attention. Consistent to this result, we found that the activation in NE\&S condition was higher than that in E\&NS condition $(t(15)=5.28$, $P<0.001$, Bonferroni corrected, $\mathrm{ES}=1.32$ ). In addition, $E \& S$ condition also showed a higher activation than E\&NS (marginally significant, $t(15)=2.88, \quad P=0.066$, Bonferroni corrected, ES $=0.72)$ and NE\&NS $(t(15)=3.32$, $P=0.025$, Bonferroni corrected, $\mathrm{ES}=0.83$ ) conditions. Finally, both V4 and LO showed significant main effects of both kinds of attention. For V4, the activation in NE\&S condition was significantly higher than that in NE\&NS condition $(t(15)=4.80, P<0.001$, Bonferroni corrected, $\mathrm{ES}=1.20$ ). Meanwhile, there was a trend that E\&NS condition showed a higher activation than NE\&NS condition $(t(15)=2.69, P=0.017$, uncorrected, $\mathrm{ES}=0.67)$. In addition, E\&S condition showed a significantly higher activation than NE\&NS condition $(t(15)=6.99, P<0.001$, Bonferroni corrected, ES=1.75). For LO, the results were similar. That is, E\&S condition $(t(15)=5.32, P<0.001$, Bonferroni corrected, ES=1.33) and NE\&S condition $(t(15)=3.69$, $P=0.012$, Bonferroni corrected, $\mathrm{ES}=0.92)$ showed significantly higher activations than NE\&NS condition. E\&NS condition showed a trend of higher activation than NE\&NS condition $(t(15)=2.35, P=0.033$, uncorrected, $\mathrm{ES}=0.59$ ). These results indicate that both emotional attention and spatial attention could boost the neural responses in V4 and LO.

We found significant modulations of emotional attention and spatial attention on the neural activities in V4 and LO. Importantly, there were no significant interactions between

Table 2 Main effects of emotional attention and spatial attention in ROls

\begin{tabular}{|c|c|c|c|c|c|c|c|c|c|c|}
\hline \multirow[t]{3}{*}{ ROls } & \multicolumn{4}{|c|}{ Activation (BOLD signal change) } & \multicolumn{6}{|c|}{ Main effect } \\
\hline & \multirow[t]{2}{*}{ E\&S } & \multirow[t]{2}{*}{ E\&NS } & \multirow[t]{2}{*}{ NE\&S } & \multirow[t]{2}{*}{ NE\&NS } & \multicolumn{3}{|l|}{ EA } & \multicolumn{3}{|l|}{ SA } \\
\hline & & & & & $F(1,15)$ & $\boldsymbol{P}$ & ES & $F(I, 15)$ & $\boldsymbol{P}$ & ES \\
\hline VI & $0.07(0.02)$ & $0.11(0.02)$ & $0.08(0.03)$ & $0.10(0.02)$ & 0.08 & 0.779 & 0.01 & 3.68 & 0.074 & 0.20 \\
\hline V2 & $0.11(0.02)$ & $0.12(0.02)$ & $0.10(0.02)$ & $0.09(0.02)$ & 3.02 & 0.010 & 0.17 & 0.19 & 0.670 & 0.01 \\
\hline V3 & $0.14(0.02)$ & $0.14(0.02)$ & $0.13(0.02)$ & $0.11(0.02)$ & 18.16 & 0.001 & 0.55 & 0.85 & 0.370 & 0.05 \\
\hline V4 & $0.23(0.02)$ & $0.19(0.03)$ & $0.22(0.02)$ & $0.14(0.01)$ & 14.99 & 0.002 & 0.50 & 58.45 & $<0.001$ & 0.80 \\
\hline IPS & $0.14(0.02)$ & $0.10(0.02)$ & $0.14(0.02)$ & $0.11(0.01)$ & 0.21 & 0.653 & 0.014 & 41.87 & $<0.001$ & 0.74 \\
\hline LO & $0.21(0.02)$ & $0.17(0.03)$ & $0.18(0.02)$ & $0.12(0.02)$ & 15.94 & 0.001 & 0.52 & 34.99 & $<0.001$ & 0.70 \\
\hline
\end{tabular}

Notes: Neural activations were averaged in the same condition across subjects and hemispheres, with standard error of mean (SEM) in parentheses. We performed a 2 (emotional attention vs no emotional attention) $\times 2$ (spatial attention vs no spatial attention) repeated measures ANOVA on the activation in each ROI. $F$ value, $P$-value and effect size (ES) of the main effects of ANOVA were reported.

Abbreviations: E\&S, contralateral to emotional picture \& spatial cue; E\&NS, contralateral to emotional picture \& no spatial cue; NE\&S, contralateral to neutral picture \& spatial cue; NE\&NS, contralateral to neutral picture \& no spatial cue; EA, emotional attention; SA, spatial attention; IPS, intraparietal sulcus; LO, lateral occipital. 


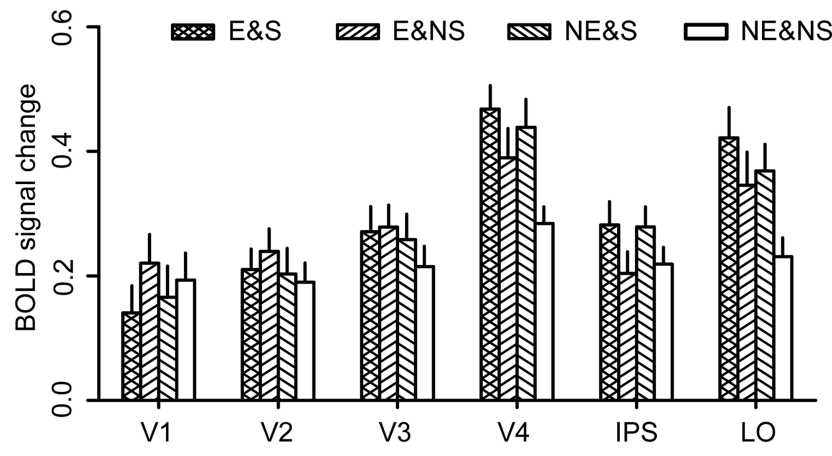

Figure 2 Obtained BOLD signal changes in conditions across subjects. Error bars denote one SEM.

Abbreviations: E\&S, contralateral to emotional picture \& spatial cue; E\&NS, contralateral to emotional picture \& no spatial cue; NE\&S, contralateral to neutral picture \& spatial cue; NE\&NS, contralateral to neutral picture \& no spatial cue; IPS, intraparietal sulcus; LO, lateral occipital.

modulations of emotional attention and spatial attention. Results from ANOVA demonstrated that the two kinds of attentional modulation weakly interacted with each other. We further elucidated it in an exploratory way, by comparing the predicted activation and the observed activation of the two kinds of attention. According to our hypothesis, if the interaction between the two kinds of attention was not evident, we may expect that the modulation of emotional attention and spatial attention (ie, E\&S - NE\&NS) should be equal to the sum of the modulations of each kind of the attention (ie, $(\mathrm{E} \& \mathrm{NS}-\mathrm{NE} \& \mathrm{NS})+(\mathrm{NE} \& \mathrm{~S}-\mathrm{NE} \& \mathrm{NS}))$. This exploratory analysis could further indicate whether the emotional attention and the spatial attention may interact with each other through examining whether the effect of the superimposed attention were the sum of the effects of the each kind of attention. After simplifying the equation, we may expect that $E \& S=E \& N S+N E \& S-N E \& N S$. Left of the equation was the observed activation of E\&S condition, while right of the equation was the predicted activation of $\mathrm{E} \& \mathrm{~S}$ condition. Then we compared the predicted activation and the observed activation of E\&S in V4 and LO (Figure 3). Paired sample $t$ tests showed no significant differences between the observed activities and predicted activities $(t(15)=-1.32, P=0.2$, $\mathrm{ES}=0.33$ for $\mathrm{V} 4$, and $t(15)=-0.84, P=0.4, \mathrm{ES}=0.21$ for $\mathrm{LO})$, which suggested that the prediction was fairly accurate and that the two kinds of attentional modulation hardly interacted with each other.

\section{Discussion}

The present study utilized a spatial cuing paradigm to measure the amplitude modulation in visual cortex, from V1, V2, V3, V4 to IPS and LO. Subjects showed a relative high and stable performance, indicating that they were

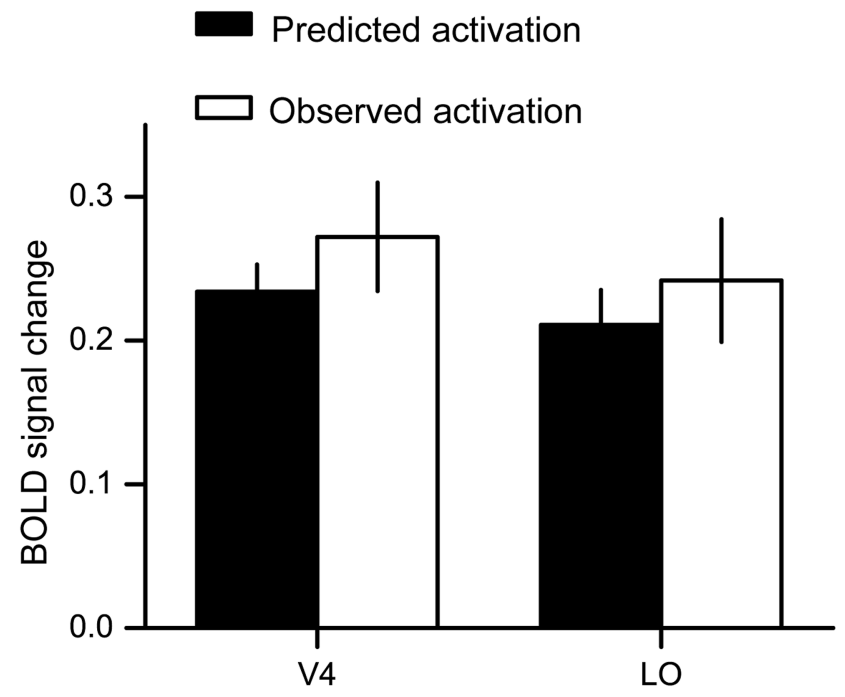

Figure 3 Results from the exploratory analysis. We compared the observed activation and predicted activation of the E\&S (contralateral to emotional picture \& spatial cue) condition. The predicted activation was calculated based on the activations of other conditions. The predictions were fairly accurate in both V4 and LO (lateral occipital). Error bars denote one SEM.

highly concentrated. The neural activation results showed significant modulation of emotional attention and spatial attention on the activities in visual cortex. Specifically, the main effect of emotional attention was significant in V3, V4 and LO, while the main effect of spatial attention was significant in V4, IPS and LO. Evidently, the activities in V4 and LO were both modulated by the emotional attention and the spatial attention, yet no significant interaction was found in any of the visual areas. Furthermore, the exploratory analysis also revealed that the interaction between modulations of spatial attention and emotional attention on the responses in V4, and LO was not significant.

The finding of significant attentional modulation by spatial cues in visual cortical areas such as V4, LO and IPS is consist with previous findings. Activities in intraparietal sulcus reflect top-down attentional control. ${ }^{40-42}$ Damage to it would lead to deficits in visual attention. ${ }^{43}$ Besides, lateral occipital complex, including V4 and LO, ${ }^{44-47}$ could be modulated by spatial attention. ${ }^{48-50}$ However, we did not found significant attentional modulation in early visual areas like V1, V2 and V3, which seems different from previous results that showed attentional modulation in primary visual cortex. ${ }^{51-53}$ One possible explanation for the negative finding in our study may be the experimental design we adopted. In our study, we used a rapid event-related design, which is limited by relatively lower signal-noise ratio (SNR) in preserving neural 
activity-induced responses. ${ }^{54}$ Furthermore, Liu et al found that transient attention did not enhance the response in primary visual cortex, which was similar to our results. ${ }^{55}$ Therefore, only strong enough modulation might be observed in the current design. As previous results showed, attentional modulation increased gradually from low-level visual areas to high-level visual areas. ${ }^{56,57}$ In another word, the attentional effect in low-level visual cortex is weak and hard to detect. It is reasonable that we only found significant modulation in the mid-level or high-level visual areas of V4 and LO.

Furthermore, our results showed that responses in V3, V4 and LO could be modulated by emotional attention. Indeed, intense emotional stimuli could induce extensive neural responses in visual cortex alone. ${ }^{58}$ Besides, previous EEG studies found that neural activities in the occipital area could be affected by emotional attention. ${ }^{21,27,59}$ Here we demonstrated that the emotional attention mainly modulated the activities in the ventral pathway. It should be noted that the emotional attention showed a much weaker effect in IPS than the effect of non-emotional spatial attention, which implied that the emotional attention may not be controlled by the IPS As we know, amygdala has a close relationship to the emotional attention. ${ }^{22}$ In addition, evidence shows that the amygdala has strong functional connections with the inferior temporal gyrus, ${ }^{28,60}$ and with the LO. ${ }^{61}$ These results and our findings together suggested that a subcortical pathway, originating from amygdala, might control emotional modulation. If it is the case, the underlying mechanisms of the emotional attention and spatial attention may be distinct. However, more evidence is needed to directly test the hypothesis.

More importantly, we examined the interaction between the two effects of emotional attention and spatial attention. No significant interaction effect was found in any of the areas including V1/V2/V3/V4/IPS and LO, which indicated that these kinds of attention may modulate the activities independently. This result was consistent to previous findings showing no interaction between the two kinds of attention in FFA. ${ }^{30}$ To further illustrate the relationship between the modulation of emotional attention and spatial attention, we performed a prediction analysis on the activities in V4 and LO. The results showed that the effect of the superimposed attention was nearly the sum of the effects of the each kind of attention. This finding further indicated that the emotional attention and the spatial attention may not interact with each other, which was consistent with previous EEG studies. ${ }^{27,62,63}$

\section{Conclusion}

In this study, we found that emotional attention and nonemotional spatial attention both exert influence on the neural activities in the human visual cortex. Importantly, these kinds of attention might have weak interaction with each other, indicating that there might be two independent mechanisms for the emotional attention and the spatial attention.

\section{Acknowledgments}

This work was supported by the New Scientist Training Program of Zunyi Meidical University ([2017]5733-014); the Scientific Research Startup Foundation of Zunyi Medical University (F-875; FB-2014-4); the National Natural Science Foundation of China (31400960); and the Guizhou province theory innovation project (Joint project, GZLCLH-2018-094).

\section{Disclosure}

The authors report no conflict of interest in this work.

\section{References}

1. Carrasco M. Visual attention: the past 25 years. Vision Res. 2011;51 (13):1484-1525. doi:10.1016/j.visres.2011.04.012

2. Posner MI. Components of visual orienting. Attention Perform $X$ Hillsdale. 1984;32(4):531-556.

3. Desimone R. Visual attention mediated by biased competition in extrastriate visual cortex. Philos Trans $R$ Soc Lond B Biol Sci. 1998;353(1373):1245-1255. doi:10.1098/rstb.1998.0280

4. Kastner S, Pinsk MA, De Weerd P, Desimone R, Ungerleider LG. Increased activity in human visual cortex during directed attention in the absence of visual stimulation. Neuron. 1999;22(4):751-761.

5. Mayo JP, Maunsell JH. Graded neuronal modulations related to visual spatial attention. $J$ Neurosci. 2016;36(19):5353-5361. doi:10.1523/JNEUROSCI.0192-16.2016

6. Carretie L, Hinojosa JA, Lopez-Martin S, Tapia M. An electrophysiological study on the interaction between emotional content and spatial frequency of visual stimuli. Neuropsychologia. 2007;45 (6):1187-1195. doi:10.1016/j.neuropsychologia.2006.10.013

7. Constantinidis C, Steinmetz MA. Neuronal responses in area $7 \mathrm{a}$ to multiple-stimulus displays: I. neurons encode the location of the salient stimulus. Cereb Cortex. 2001;11(7):581-591.

8. Downar J, Crawley AP, Mikulis DJ, Davis KD. A cortical network sensitive to stimulus salience in a neutral behavioral context across multiple sensory modalities. J Neurophysiol. 2002;87(1):615-620. doi:10.1152/jn.00636.2001

9. Shulman GL, Astafiev SV, Franke D, et al. Interaction of stimulusdriven reorienting and expectation in ventral and dorsal frontoparietal and basal ganglia-cortical networks. J Neurosci. 2009;29(14):43924407. doi:10.1523/JNEUROSCI.5609-08.2009

10. Corbetta M, Shulman GL. Human cortical mechanisms of visual attention during orienting and search. Philos Trans R Soc Lond B Biol Sci. 1998;353(1373):1353-1362. doi:10.1098/rstb.1998.0289

11. Kastner S, Ungerleider LG. Mechanisms of visual attention in the human cortex. Annu Rev Neurosci. 2000;23:315-341. doi:10.1146/ annurev.neuro.23.1.315 
12. Desimone R, Duncan J. Neural mechanisms of selective visual attention. Annu Rev Neurosci. 1995;18:193-222. doi:10.1146/annurev. ne.18.030195.001205

13. Galvez-Pol A, Calvo-Merino B, Capilla A, Forster B. Persistent recruitment of somatosensory cortex during active maintenance of hand images in working memory. Neuroimage. 2018;174:153-163. doi:10.1016/j.neuroimage.2018.03.024

14. Galvez-Pol A, Forster B, Calvo-Merino B. Modulation of motor cortex activity in a visual working memory task of hand images. Neuropsychologia. 2018;117:75-83. doi:10.1016/j. neuropsychologia.2018.05.005

15. Martinez A, Anllo-Vento L, Sereno MI, et al. Involvement of striate and extrastriate visual cortical areas in spatial attention. Nat Neurosci. 1999;2(4):364-369. doi:10.1038/7274

16. Squire RF, Noudoost B, Schafer RJ, Moore T. Prefrontal contributions to visual selective attention. Annu Rev Neurosci. 2013;36:451466. doi:10.1146/annurev-neuro-062111-150439

17. Corbetta M, Shulman GL. Control of goal-directed and stimulusdriven attention in the brain. Nat Rev Neurosci. 2002;3(3):201-215. doi:10.1038/nrn755

18. Frischen A, Eastwood JD, Smilek D. Visual search for faces with emotional expressions. Psychol Bull. 2008;134(5):662-676. doi:10.1037/0033-2909.134.5.662

19. Maratos FA, Senior C, Mogg K, Bradley BP, Rippon G. Early gamma-band activity as a function of threat processing in the extrastriate visual cortex. Cogn Neurosci. 2012;3(1):62-68. doi:10.1080/ 17588928.2011.613989

20. Pourtois G, Grandjean D, Sander D, Vuilleumier P. Electrophysiological correlates of rapid spatial orienting towards fearful faces. Cereb Cortex. 2004;14(6):619-633. doi:10.1093/cercor/bhh023

21. Vuilleumier P, Driver J. Modulation of visual processing by attention and emotion: windows on causal interactions between human brain regions. Philos Trans R Soc Lond B Biol Sci. 2007;362(1481):837855. doi:10.1098/rstb.2007.2092

22. Vuilleumier P. How brains beware: neural mechanisms of emotional attention. Trends Cogn Sci. 2005;9(12):585-594. doi:10.1016/j. tics.2005.10.011

23. Carretie L. Exogenous (automatic) attention to emotional stimuli: a review. Cogn Affect Behav Neurosci. 2014;14(4):1228-1258. doi:10.3758/s13415-014-0270-2

24. de Gelder B, Hortensius R, Tamietto M. Attention and awareness each influence amygdala activity for dynamic bodily expressions - a short review. Front Integr Neurosci. 2012;6:54. doi:10.3389/ fnint.2012.00054

25. Öhman A. Automaticity and the amygdala: nonconscious responses to emotional faces. Curr Dir Psychol Sci. 2002;11(2):62-66. doi:10.1111/1467-8721.00169

26. Pourtois G, Schettino A, Vuilleumier P. Brain mechanisms for emotional influences on perception and attention: what is magic and what is not. Biol Psychol. 2013;92(3):492-512. doi:10.1016/j. biopsycho.2012.02.007

27. Keil A, Moratti S, Sabatinelli D, Bradley MM, Lang PJ. Additive effects of emotional content and spatial selective attention on electrocortical facilitation. Cereb Cortex. 2005;15(8):1187-1197. doi:10.1093/cercor/bhi001

28. Wendt J, Weike AI, Lotze M, Hamm AO. The functional connectivity between amygdala and extrastriate visual cortex activity during emotional picture processing depends on stimulus novelty. Biol Psychol. 2011;86(3):203-209. doi:10.1016/j.biopsycho.2010.11.009

29. Pessoa L, Kastner S, Ungerleider LG. Attentional control of the processing of neural and emotional stimuli. Brain Res Cogn Brain Res. 2002;15(1):31-45.

30. Vuilleumier P, Armony JL, Driver J, Dolan RJ. Effects of attention and emotion on face processing in the human brain: an event-related fMRI study. Neuron. 2001;30(3):829-841.
31. Theeuwes J. Top-down and bottom-up control of visual selection. Acta Psychol (Amst). 2010;135(2):77-99. doi:10.1016/j. actpsy.2010.02.006

32. Fichtenholtz HM, Hopfinger JB, Graham R, Detwiler JM, LaBar KS. Event-related potentials reveal temporal staging of dynamic facial expression and gaze shift effects on attentional orienting. Soc Neurosci. 2009;4(4):317-331. doi:10.1080/17470910902809487

33. Engel SA, Glover GH, Wandell BA. Retinotopic organization in human visual cortex and the spatial precision of functional MRI. Cereb Cortex. 1997;7(2):181-192.

34. Sereno MI, Dale AM, Reppas JB, et al. Borders of multiple visual areas in humans revealed by functional magnetic resonance imaging. Science. 1995;268(5212):889-893.

35. DeYoe EA, Carman GJ, Bandettini P, et al. Mapping striate and extrastriate visual areas in human cerebral cortex. Proc Natl Acad Sci U S A. 1996;93(6):2382-2386.

36. Slotnick SD, Yantis S. Efficient acquisition of human retinotopic maps. Hum Brain Mapp. 2003;18(1):22-29. doi:10.1002/hbm.10077

37. Wade AR, Brewer AA, Rieger JW, Wandell BA. Functional measurements of human ventral occipital cortex: retinotopy and colour. Philos Trans $R$ Soc Lond B Biol Sci. 2002;357(1424):963-973. doi:10.1098/rstb.2002.1108

38. Smith AM, Lewis BK, Ruttimann UE, et al. Investigation of low frequency drift in fMRI signal. Neuroimage. 1999;9(5):526-533. doi:10.1006/nimg.1999.0435

39. Kourtzi Z, Kanwisher N. Activation in human MT/MST by static images with implied motion. J Cogn Neurosci. 2000;12(1):48-55.

40. Coull JT, Frith CD. Differential activation of right superior parietal cortex and intraparietal sulcus by spatial and nonspatial attention. Neuroimage. 1998;8(2):176-187. doi:10.1006/nimg.1998.0354

41. Schultz J, Lennert T. BOLD signal in intraparietal sulcus covaries with magnitude of implicitly driven attention shifts. Neuroimage. 2009;45(4):1314-1328. doi:10.1016/j.neuroimage.2009.01.012

42. Silk TJ, Bellgrove MA, Wrafter P, Mattingley JB, Cunnington R. Spatial working memory and spatial attention rely on common neural processes in the intraparietal sulcus. Neuroimage. 2010;53(2):718724. doi:10.1016/j.neuroimage.2010.06.068

43. Gillebert CR, Mantini D, Thijs V, Sunaert S, Dupont P, Vandenberghe R. Lesion evidence for the critical role of the intraparietal sulcus in spatial attention. Brain. 2011;134(Pt 6):1694-1709. doi:10.1093/ brain/awr085

44. Cant JS, Goodale MA. Attention to form or surface properties modulates different regions of human occipitotemporal cortex. Cereb Cortex. 2007;17(3):713-731. doi:10.1093/cercor/bhk022

45. Connor CE, Preddie DC, Gallant JL, Van Essen DC. Spatial attention effects in macaque area V4. J Neurosci. 1997;17(9):3201-3214.

46. Niemeier M, Goltz HC, Kuchinad A, Tweed DB, Vilis T. A contralateral preference in the lateral occipital area: sensory and attentional mechanisms. Cereb Cortex. 2005;15(3):325-331. doi:10.1093/cercor/ bhh134

47. Roe AW, Chelazzi L, Connor CE, et al. Toward a unified theory of visual area V4. Neuron. 2012;74(1):12-29. doi:10.1016/j. neuron.2012.03.011

48. Large ME, Aldcroft A, Vilis T. Task-related laterality effects in the lateral occipital complex. Brain Res. 2007;1128(1):130-138. doi:10.1016/j.brainres.2006.10.023

49. Murray SO, Wojciulik E. Attention increases neural selectivity in the human lateral occipital complex. Nat Neurosci. 2004;7(1):70-74. doi:10.1038/nn1161

50. Scholte HS, Sligte I, Groen I, Lamme V, Ghebreab S. The posterior part of the lateral occipital complex analyzes the spatial correlation structure of natural visual scenes. $J$ Vis. 2013;13(9):1098. doi:10.1167/13.9.1098

51. Gandhi SP, Heeger DJ, Boynton GM. Spatial attention affects brain activity in human primary visual cortex. Proc Natl Acad Sci U S A. 1999;96(6):3314-3319. 
52. Somers DC, Dale AM, Seiffert AE, Tootell RB. Functional MRI reveals spatially specific attentional modulation in human primary visual cortex. Proc Natl Acad Sci U S A. 1999;96(4):1663-1668.

53. Watanabe T, Sasaki Y, Miyauchi S, et al. Attention-regulated activity in human primary visual cortex. J Neurophysiol. 1998;79(4):22182221. doi:10.1152/jn.1998.79.4.2218

54. LaConte SM, Ngan SC, Hu X. Wavelet transform-based Wiener filtering of event-related fMRI data. Magn Reson Med. 2000;44 (5):746-757. doi:10.1002/1522-2594(200011)44:5<746::AIDMRM13>3.0.CO;2-O

55. Liu T, Pestilli F, Carrasco M. Transient attention enhances perceptual performance and FMRI response in human visual cortex. Neuron 2005;45(3):469-477. doi:10.1016/j.neuron.2004.12.039

56. Moran J, Desimone R. Selective attention gates visual processing in the extrastriate cortex. Science. 1985;229(4715):782-784.

57. Reynolds JH, Chelazzi L, Desimone R. Competitive mechanisms subserve attention in macaque areas V2 and V4. J Neurosci. 1999;19(5):1736-1753.

58. Bradley MM, Sabatinelli D, Lang PJ, Fitzsimmons JR, King W, Desai P. Activation of the visual cortex in motivated attention. Behav Neurosci. 2003;117(2):369-380.
59. Gomez A, Rothkirch M, Kaul C, et al. Emotion modulates the effects of endogenous attention on retinotopic visual processing. Neuroimage. 2011;57(4):1542-1551. doi:10.1016/j. neuroimage.2011.05.072

60. Sabatinelli D, Bradley MM, Fitzsimmons JR, Lang PJ. Parallel amygdala and inferotemporal activation reflect emotional intensity and fear relevance. Neuroimage. 2005;24(4):1265-1270. doi:10.1016/j.neuroimage.2004.12.015

61. Rotshtein P, Malach R, Hadar U, Graif M, Hendler T. Feeling or features: different sensitivity to emotion in high-order visual cortex and amygdala. Neuron. 2001;32(4):747-757.

62. Brosch T, Pourtois G, Sander D, Vuilleumier P. Additive effects of emotional, endogenous, and exogenous attention: behavioral and electrophysiological evidence. Neuropsychologia. 2011;49(7):17791787. doi:10.1016/j.neuropsychologia.2011.02.056

63. Hietanen JK, Kirjavainen I, Nummenmaa L. Additive effects of affective arousal and top-down attention on the event-related brain responses to human bodies. Biol Psychol. 2014;103:167-175. doi:10.1016/j.biopsycho.2014.09.003 


\section{Supplementary material}

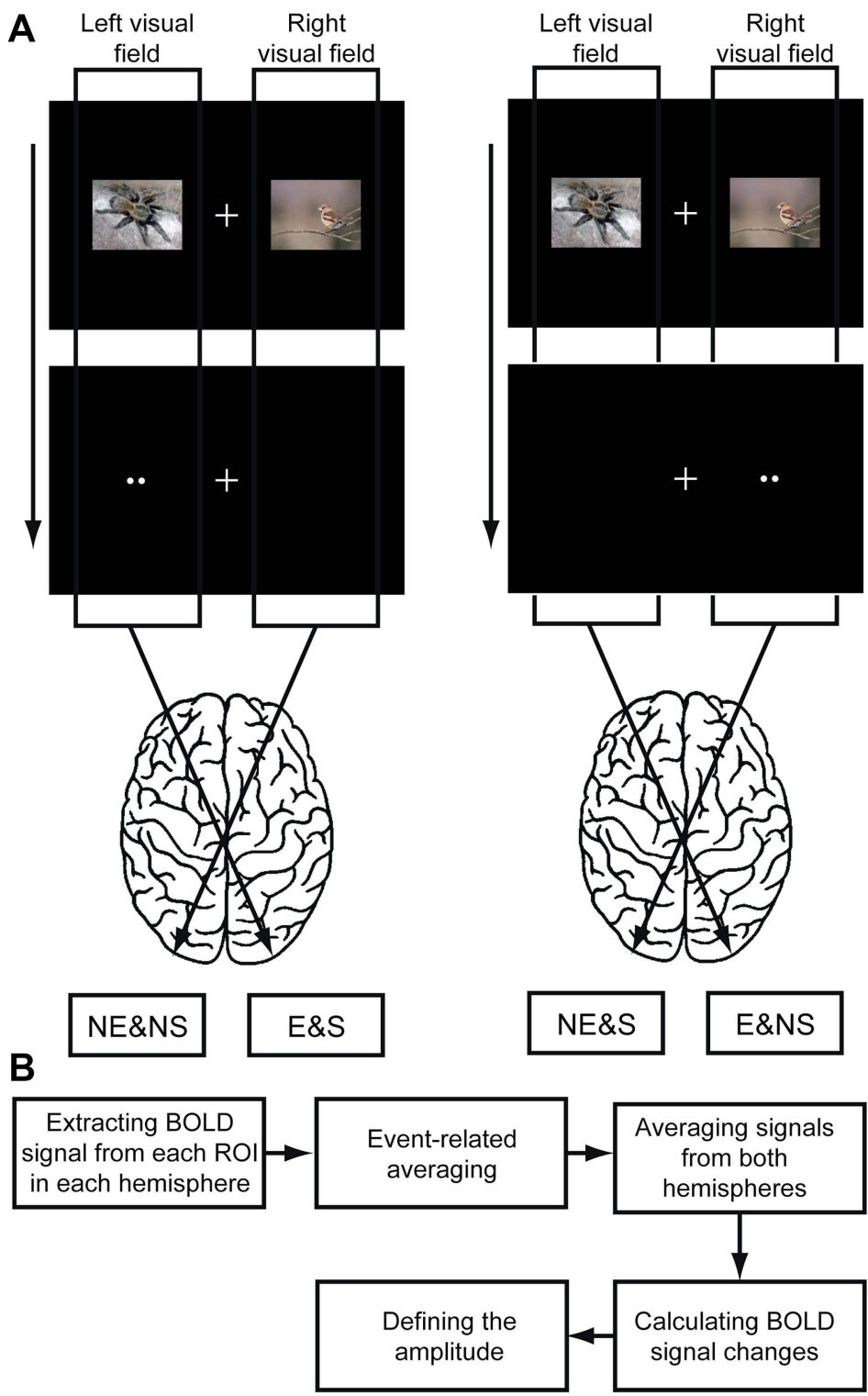

Figure SI (A) Demonstration of the setting of conditions. (B) The procedure of fMRI data analysis after preprocessing the functional images.

Abbreviations: E\&S, contralateral to emotional picture \& spatial cue; E\&NS, contralateral to emotional picture \& no spatial cue; NE\&S, contralateral to neutral picture \& spatial cue; NE\&NS, contralateral to neutral picture \& no spatial cue.

Psychology Research and Behavior Management

\section{Publish your work in this journal}

Psychology Research and Behavior Management is an international, peer-reviewed, open access journal focusing on the science of psychology and its application in behavior management to develop improved outcomes in the clinical, educational, sports and business arenas. Specific topics covered in the journal include: Neuroscience, memory and decision making; Behavior modification and management; Clinical applications; Business and sports performance management; Social and developmental studies; Animal studies. The manuscript management system is completely online and includes a very quick and fair peer-review system, which is all easy to use. Visit http://www. dovepress.com/testimonials.php to read real quotes from published authors. 